\title{
TIME-DOMAIN FLUORESCENCE LIFETIME IMAGING APPLIED TO BIOLOGICAL TISSUE
}

\author{
Dan Elson, Jose Requejo-Isidro, Ian Munro, Fred Reavell, Jan Siegel, Klaus Suhling, Paul Tadrous, \\ Richard Benninger, Peter Lanigan, James McGinty, Clifford Talbot, Bebhinn Treanor, Stephen Webb, \\ Ann Sandison, Andrew Wallace, Dan Davis, John Lever, Mark Neil, David Phillips, Gordon Stamp, and \\ Paul French
}

Photonics Group, Physics Department, Imperial College London, London SW7 2AZ, U.K. ds.elson@imperial.ac.uk

\begin{abstract}
Fluorescence lifetime imaging (FLIM) is a functional imaging methodology that can provide information, not only concerning the localisation of specific fluorophores, but also about the local fluorophore environment. It may be implemented in scanning confocal or multi-photon microscopes, or in wide-field microscopes and endoscopes. When applied to tissue autofluorescence, it reveals intrinsic excellent contrast between different types and states of tissue. This article aims to review our recent progress in developing time-domain FLIM technology for microscopy and endoscopy and applying it to biological tissue.

OCIS codes: (170.2520) Fluorescence microscopy; (170.3650) Lifetime based sensing; (170.6920) Time resolved imaging
\end{abstract}

\section{Introduction}

Fluorescence lifetime imaging (FLIM) is a functional imaging methodology that is currently experiencing a rapid increase in uptake due to its excellent specificity and its relative insensitivity to intensity artefacts. FLIM is applicable to imaging intracellular function, e.g. using the Förster resonance energy transfer (FRET) technique and can provide information, not only concerning the localisation of a specific fluorophore, but also about the local fluorophore environment (e.g. oxygen, $\left[\mathrm{Ca}^{2+}\right], \quad \mathrm{pH}$ etc [1]). It may be implemented in scanning confocal or multi-photon microscopes, as well as in wide-field microscopes and endoscopes. When applied to tissue autofluorescence, it reveals intrinsic contrast between different types and states of tissue, potentially providing new non-invasive functional/diagnostic imaging modalities [2]. This paper aims to describe our approach to timedomain FLIM and to review our recent work, as presented at the ESP 2003 conference [3] in Vienna, with particular emphasis on its application to biological tissue. It is not intended as a general discussion of FLIM - for this we could suggest the papers by Cubeddu et al. [4], Wouters et al. [5] and Tadrous [6]. For a discussion of FLIM applied to FRET, we suggest the paper by Bastiaens and Squire [7].

Fluorescence imaging is particularly powerful since the use of fluorescent labels can yield high specificity, while quantitative analysis of the fluorescence signal can provide information about the local environment of the fluorophore molecules as well as their localisation. The fluorescence quantum efficiency, $\eta$, defined as the number of fluorescence photons emitted per excitation photon absorbed, is given by $\Gamma /(\Gamma+k)$, where $\Gamma$ and $k$ are the radiative and non-radiative decay rates respectively. In general, the radiative decay rate is a characteristic of the fluorophore molecule, related to the strength of the dipole moment, while the non-radiative decay rate can vary according to how the fluorophore interacts with its local environment. For some fluorophores, $k$ is sensitive to physical factors such as the local viscosity, or to chemical factors such as the local $\mathrm{pH}$ or to calcium ion concentration. Thus fluorescence "probes" may be employed to produce functional maps of perturbations of such environmental factors by inferring changes in quantum efficiency from fluorescence intensity measurements. In heterogeneous biological samples, however, it can be difficult to determine (variations in) the quantum efficiency owing to possible artefacts arising from non-uniform excitation flux, fluorophore concentration and/or detection efficiency. Optical scattering in thick biological samples can also degrade quantitative intensity measurements. A more robust approach is to make relative intensity measurements. One approach is to employ special probes whose fluorescence spectra change in a predictable way according to the strength of the local environmental perturbation (e.g. $\left.\left[\mathrm{Ca}^{2+}\right]\right)$. By incorporating these probes into a sample and spectrally resolving fluorescence intensity measurements at two or more wavelengths, it is possible to produce a map of, e.g. $\left[\mathrm{Ca}^{2+}\right]$ distributions to study the operation of nerve synapses. Such spectrally-resolved relative measurements cancel out factors due to optical scattering, as well as non-uniformity in fluorophore concentration, excitation and detection efficiency. This "wavelength-ratiometric imaging" 
has been applied to microscopy and to in vivo diagnostic imaging [8]. Unfortunately, there are a limited number of experimental situations for which suitable wavelength-ratiometric probes (or endogenous fluorophores) are available.

An alternative approach to acquiring functional information from relative intensity measurements (or images) is to temporally resolve the fluorescence profiles. FLIM entails determining the (average) fluorescence decay time for each pixel in a field of view and producing a map of fluorescence lifetime data. Like the quantum efficiency, $\eta$, the fluorescence lifetime $(\tau=1 /(\Gamma+k)$,) also depends on the radiative and non-radiative decay rates and, like $\eta$, can be used to contrast different fluorophore species (via $\Gamma$ ) and different local fluorophore environments (via $k$ ). FLIM is being applied to biological samples ranging from single cells to bulk tissue using both exogenous fluorophore labels, particularly genetically expressed labels such as green fluorescent protein, and endogenous fluorophores in biological tissue to provide intrinsic contrast. Increasingly FLIM and related techniques are being applied to microanalysis of sample arrays and high throughput screening (HTS).

\section{Technology for FLIM}

Most biologically relevant fluorophores exhibit characteristic decay times ranging from picoseconds to nanoseconds. For example, enhanced green fluorescent protein (EGFP) typically decays with both ps and ns components and many endogenous tissue fluorophores fall within this range, e.g. [9]. Imaging with such time resolution has presented a significant technological barrier for FLIM to find widespread adoption. Until recently, the lack of commercially available FLIM instrumentation and the significant technological expertise required to develop such instrumentation in-house has precluded application in the life sciences for all but a few specialist groups. Ultrafast laser technology, however, has become increasingly user-friendly - if not increasingly cheap - and the recent proliferation of multi-photon microscopes provides a straightforward route to implement FLIM using commercially available off-the shelf components. Since multi-photon microscopes inherently acquire images scanning pixel by pixel and already incorporate an ultrashort-pulsed excitation laser, it is relatively simple to add time-correlated single photon counting (TCSPC), e.g. [10], to the detection channel and obtain time-resolved fluorescence decay profiles for each pixel, e.g. [11]. Multi-photon imaging also provides inherent optical sectioning and is particularly effective in highly scattering samples such as living tissue. It is ironic, however, that this "add-on" option to multi-photon scanning microscopes, which is arguably the most technologically complex and expensive approach to obtain fluorescence lifetime images, has probably become the most widespread. Recent developments in picosecond pulsed diode lasers, however, offer a considerably cheaper alternative when combined with TCSPC in singlephoton confocal microscopes, e.g. [12] to provide superior 3-D spatial resolution and higher fluorescence signal levels - leading to higher imaging rates.

An alternative implementation of single channel FLIM uses a frequency domain approach, in which synchronous detection is used to determine the phase difference and change in modulation depth between a sinusoidally modulated excitation laser signal and the resulting sinusoidally modulated fluorescence signal. This has been implemented in wide-field microscopy, e.g. [13, 14] and confocal scanning microscopy, e.g. [15]. This "lock-in" detection scheme may also be employed using a pulsed source and has been implemented in multiphoton microscopy, e.g. [16]. In principle the time and frequency approaches can provide equivalent information but in practice the choice of approach is impacted by the specific FLIM application. The frequency domain approach is sometimes considered to be simpler and cheaper since it is straightforward to sinusoidally modulate a c.w. laser for nanosecond measurements. This approach has been very successfully applied to wide-field imaging of FRET, e.g. [17]. When picosecond resolution is required, the necessary high frequency $(\mathrm{GHz})$ electronics means that the cost and complexity of the frequency domain technology becomes comparable to that of the time domain. A further consideration is that biological systems often exhibit complex fluorescence decays that do not fit to a simple mono-exponential decay model. While double exponential decay profiles may be readily analysed in the frequency domain, more complex decay profiles - particularly those corresponding to a continuous spectrum of lifetime components [18] - may be more straightforward to analyse from direct time-domain measurements.

Apart from cost and complexity, the main drawback to FLIM using confocal/multiphoton scanning microscopes is that it can be slow, since the fluorescence signals from each pixel are analysed sequentially. Many biological fluorophores do exhibit complex exponential fluorescence decay profiles and these can require long integration times per pixel to achieve a sufficient signal to noise ratio to provide reliable curve fitting. Similar considerations apply to spectrally resolved imaging - generally it is inevitable that the more information that is 
required (i.e. bits/pixel), the more photons must be collected and the longer will be the data acquisition time for the whole image. Extended pixel dwell times and/or high intensities can lead to problems associated with photobleaching, although this may be ameliorated for many samples by multi-photon excitation. It seems attractive therefore to interrogate all the sample pixels in parallel when undertaking functional imaging, in order to minimise the image acquisition time, reduce photobleaching and facilitate the observation of rapidly evolving systems. Thus wide-field FLIM - as well as widefield multi-spectral imaging - offers advantages for biological and medical applications beyond their reduced cost and complexity compared to confocal scanning instrumentation.

\section{Wide-field FLIM}

Before the availability of low cost, high speed TCSPC cards, FLIM was most commonly realised in wide-field microscopes using either timedomain or frequency domain detection. Figure 1 shows a schematic of wide-field time domain FLIM, in which time-gated "snapshots" of the fluorescence emission are acquired at various nanosecond delays after the excitation - with $\sim 100$ ps shutter speeds, usually achieved using high speed gated optical image intensifiers (GOI's), e.g. [19]. This series of time-gated fluorescence intensity images can then be analysed to extract the fluorescence decay time for each pixel of the image. Although such time-domain FLIM systems do require ultrafast lasers, making them expensive and complex, we have demonstrated at Imperial College London that they may be implemented using diode-pumped solid-state laser technology [20] or even compact and inexpensive pulsed diode lasers [21]. Figure 2 shows examples of wide-field FLIM applied to arrays of dye samples in multi-well plates. These lifetime maps were obtained using a pulsed diode laser (PicoQuant $\mathrm{GmbH})$ and a gated optical image intensifier (Kentech Instruments Ltd). Figure 2(a) illustrates FLIM contrasting different molecular species while figure 2(b) shows fluorescence lifetime contrasting changes in the local fluorophore environment (solvent viscosity varied by adjusting a solvent mixture of ethanol and glycerol) for the same molecular species.

Wide-field frequency domain FLIM is also usually implemented using an optical image intensifier: in this case a sinusoidally modulated gain provides wide- field synchronous detection in conjunction with a sinusoidally modulated excitation beam [13, 14]. As with single channel frequency domain lifetime measurements [22], it should be possible to use pulsed excitation sources to provide multiple excitation frequencies for imaging of complex fluorescence decay profiles, which may be analysed at multiple frequencies simultaneously [23]. There is also the intriguing approach to directly modulate the gain of a CCD camera to realise frequency domain FLIM [24].

One drawback of wide-field, compared to confocal/multi-photon scanning FLIM, is that one loses the inherent optical sectioning of such microscopes. There have, however, been a number of approaches to realise wide-field optical sectioning and these may all be combined with wide-field FLIM. One of the most established approaches is tandem scanning confocal microscopy [25], which uses an array of pinholes to interrogate many pixels in parallel. This approach may be adapted to multi-foci multiphoton microscopy, for which an array of parallel (near infrared) excitation beams is rapidly scanned across the focal plane to create a sectioned fluorescence image suitable for wide-field detection [26]. An experimentally simpler approach is that of structured illumination, which computes a wide-field sectioned image and a conventional wide-field image from three acquisitions of spatially modulated images [27]. We have combined structured illumination with FLIM [28] and also combined this with multispectral imaging to demonstrate wide-field 5-D fluorescence imaging for the first time [29]. Such multi-dimensional and potentially high-speed 
fluorescence imaging could provide powerful tools for assay technology and clinical diagnosis, as well for molecular imaging of live cell dynamics.

\section{FLIM microscopy applied to investigate fluorophore environment}

Because FLIM can provide information about the local fluorophore environment, as well as the localisation of a fluorescent label or labelled protein, it offers the potential to study intracellar structure and function using appropriate fluorophores. For example, figure 3(a) illustrates a multi-well plate FLIM experiment in which a variation in the local solvent refractive index of recombinant enhanced green fluorescence protein (EGFP) solution produces a reproducible change in fluorescence lifetime [30] that, in this case, results from the refractive index dependence of the radiative decay rate [31]. We are interested to investigate the potential of probing the local environment of EGFP-tagged proteins in cells. Figure 3(b) shows a (multiphoton TCSPC) FLIM image of human B cells with EGFP-tagged MHC protein interacting with an unlabelled NK cell and forming an "immune synapse" [32]. It is interesting to consider the apparent heterogeneity often observed in such fluorescence lifetime images. By comparing the observed distributions of (fitted) lifetime values with the statistical variation expected as a consequence of the fluctuations in fluorescence intensity across the image, we conclude that the observed variation in fluorescence lifetime observed in the cell membrane in figure $3(\mathrm{~b})$ is not likely to be significant but the lifetime difference observed at the "immune synapse" between the B cell and the NK cell appears to be "real" [33]. At present the origin of this lifetime difference at the immune synapse is not known. We speculate that it may indicate a change in the local refractive index, although other explanations are possible and further study is required to clarify this issue. Figure 3(c) shows a view of a 3-D FLIM stack of a similar B cell in which a lifetime change at the NK cell synapse is also apparent. The full potential for intracellular FLIM is yet to be realised but there may be opportunities to correlate variations in local fluorophore environment with different cell constituents, e.g. lipids, proteins etc and to investigate the dynamics of intercellular and intracellular processes. Equally there is scope to use FLIM with other fluorophore labels (genetically expressed and otherwise) to study biological function - and to obtain appropriate biological contrast for assays. FLIM is frequently applied to imaging bimolecular interactions such as Förster resonance energy transfer (FRET), which reports on the distance between a donor and acceptor molecule, e.g. [34], [35]. FLIM-FRET is

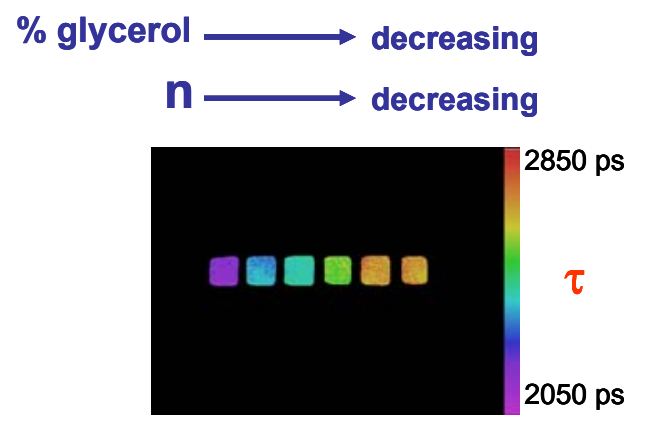

(a)

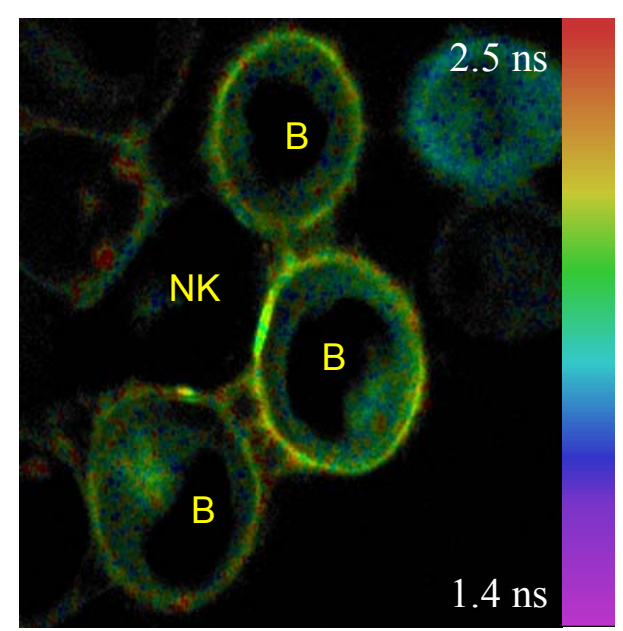

(b)

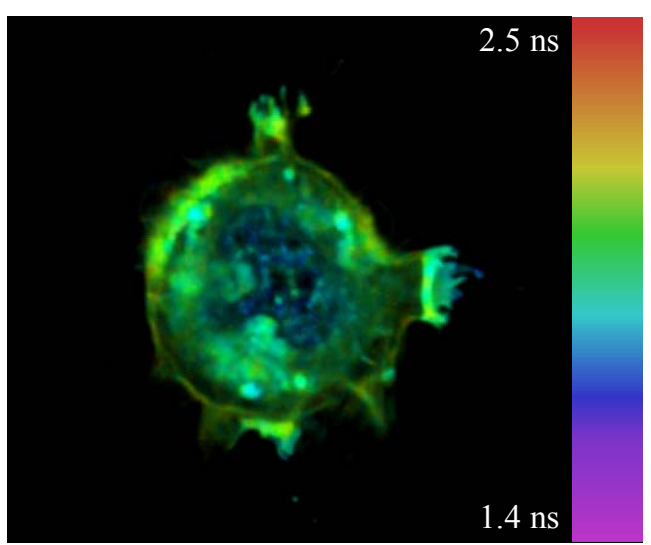

(c)

Figure 3. (a) Multi-well plate array of EGFP solutions of varying refractive index [from reference [30], (b) sectioned multiphoton TCPSC FLIM image and (c) picture of $3 D$ FLIM stack of B cells with EGFP-tagged MHC protein at immune synapse with an NK cell

attractive compared to intensity-based imaging techniques because the fluorescence decay profiles depend on the excited state reactions but not on fluorophore concentration or optical path length [7]. A particular opportunity provided by FLIM is when the donor and acceptor emission overlap 
such that they cannot be spectrally distinguished [36].

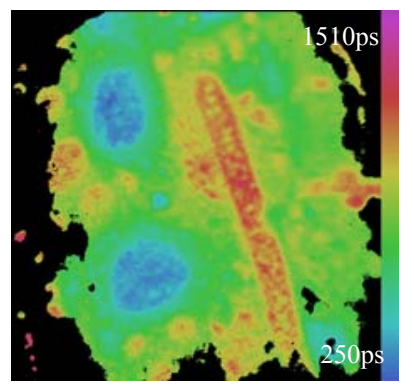

(a)

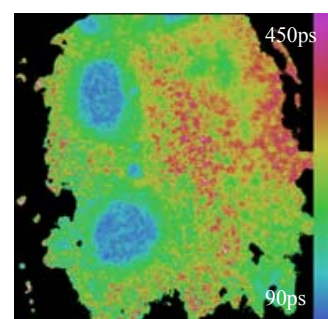

(b)

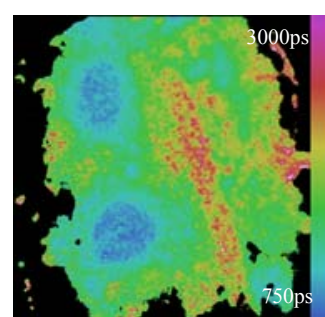

(c)
Stretched exponential function

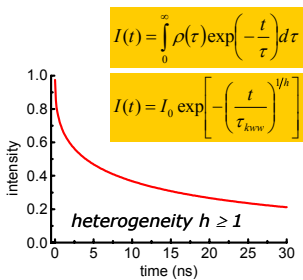

Continuous lifetime distribution $\rho(\tau)$

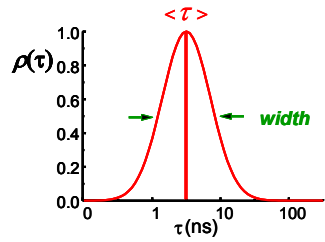

Plotting parameters

- average lifetime $\langle\tau\rangle=h \cdot \tau_{\kappa w w} \cdot \Gamma(h)$

- heterogeneity $h \sim$ width

(d)

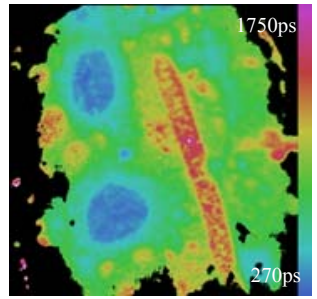

(e)

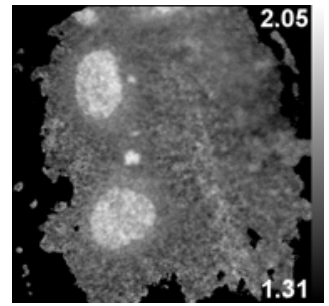

(f)
Figure 4. FLIM maps of autofluorescence of an unstained section of rat ear showing elastic cartilage, blood vessels and background collagen: (a) corresponds to a single exponential decay model; $(b, c)$ correspond to the first and second discrete lifetime components respectively of a double exponential decay model; (d) is a representation of the stretched exponential function and $(e, f)$ are the mean lifetime and heterogeneity maps obtained using the stretched exponential model (from reference [18])
Endogenous FLIM for intrinsic tissue contrast Imaging tissue autofluorescence may provide powerful tools for non-invasive clinical diagnosis. References [37] and [38] provide recent reviews of tissue spectroscopy. Although intensity and spectrally resolved imaging have been widely applied to tissue autofluorescence, the origin of observed fluorescence contrast is still not fully understood and, to date, there has been relatively little FLIM of tissue autofluorescence. Most timeresolved spectroscopic studies of biological tissue have used nitrogen lasers or nitrogen laser-pumped dye lasers and much of the published tissue lifetime data is for ultraviolet excitation, e.g. $[39,40]$. Our work towards the development of clinical FLIM instrumentation has mainly utilised frequency-doubled mode-locked Cr:LiSAF and Ti:Sapphire lasers to provide excitation wavelengths above $400 \mathrm{~nm}$, for which FLIM has been shown to provide intrinsic label-free contrast in bulk tissue, e.g. [19]. Figure 4 shows wide-field time domain FLIM maps of unstained rat tissue obtained using picosecond excitation pulses at 410 $\mathrm{nm}[18]$.

Figure 4(a) is a FLIM map generated assuming a single exponential decay for all pixels. Although it contrasts the elastic cartilage, blood vessel walls, clotted blood and the background of collagen, analysis of the fitted parameters reveals that most of the pixels do not report a single exponential fluorescence decay profile. A common strategy for dealing with complex fluorescence decay profiles is to fit multiple exponential decay components. The concomitant increase in the number of fitting parameters will improve the reported quality of fit but the resulting FLIM maps will tend to appear noisier, as shown in figure $4(\mathrm{~b}, \mathrm{c})$. The assumption of a discrete number of lifetime components in biological tissue is somewhat arbitrary. Given that the tissue components are substantially smaller than the pixel resolution element, it is possible (and likely) that the intrinsic fluorescence will report a distribution of similar but not identical fluorescent species within each sample pixel. We have investigated the use of the stretched exponential decay profile and have found this approach to provide superior lifetime images, in significantly less computation time, than fitting to a double exponential decay model [10]. Figure 4(d) shows a stretched exponential decay profile together with the corresponding continuous lifetime distribution. Figure 4(e) shows a map of the mean fluorescence lifetime for the same data set used for figure 4. As described in [18], the mean lifetime map is obtained by fitting the acquired time-gated fluorescence intensity images to the stretched exponential function to obtain values for $\mathrm{h}$ and $\tau_{\mathrm{kww}}$. The expression to calculate the mean lifetime is derived by integrating the 
equation for the stretched exponential decay function. Figure 4(f) shows a plot of the heterogeneity parameter, $\mathrm{h}$, which is related to the width of the lifetime distribution for each pixel and which provides a further opportunity for intrinsic contrast. In this case the heterogeneous blood is

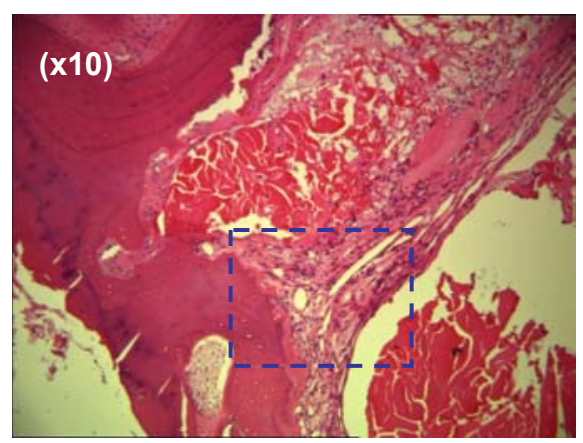

(a)

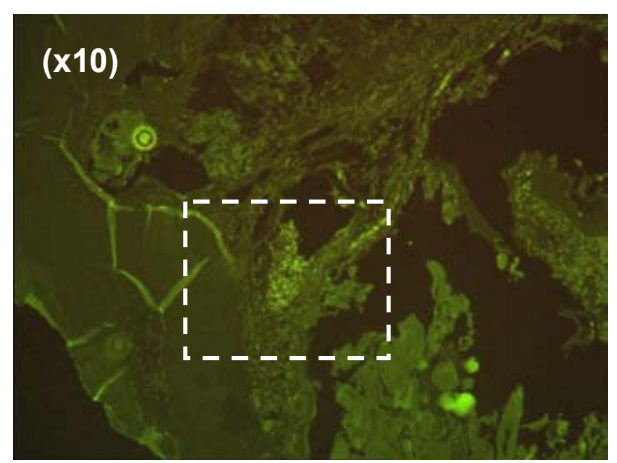

(b)

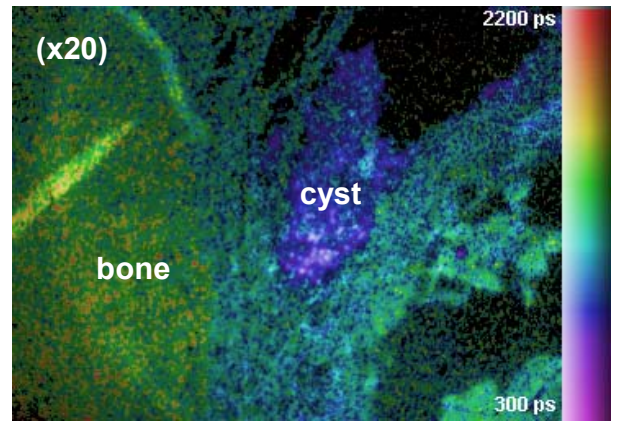

(c)

Figure 5. (a) H\&E stained section, (b) wide-field fluorescence image and (c) wide-field FLIM image of unstained section of fixed human femoral head, showing bone, cartilage and subchondral cyst formation. (FLIM map colour scale is from $300-$ $2200 \mathrm{ps})$

contrasted with the more homogeneous connective tissue. By taking the Laplace transform of the experimental complex fluorescence decay profiles in this data set, we have shown that they do indeed correspond to a continuous lifetime distribution []. We consider that, unless a priori knowledge suggests multiple discrete exponential decay components, e.g. in the case of specific fluorescent labels, it is empirically more effective to assume a decay model corresponding to continuous lifetime distributions and, for biological tissue, likely to be a more accurate representation of the underlying physiology.

Compared to a single exponential decay, more complex fluorescence decay profiles such as the stretched exponential and multi-exponential models inevitably require significantly more data to be acquired and are more vulnerable to noise artefacts - particularly relating to uncertainty in the background level and the temporal instrument response function. Given the current state of FLIM technology, practical FLIM systems applied to tissue imaging are usually restricted to the assumption of mono-exponential fluorescence decay profiles. Figure 4 illustrates that this approach does provide much useful contrast with the benefits of faster image acquisition and computation. Our initial wide-field time domain FLIM experiments have followed this approach and we have investigated a number of different fixed and frozen ex vivo tissue samples with a view to developing clinical instrumentation based on intrinsic autofluorescence contrast.

Figure 5 shows an example of intrinsic FLIM contrast in fixed sections of (human) femoral head that indicate marked degeneration and loss of articular cartilage with collapse of subchondral bone, and subchondral cyst formation. These FLIM images, which indicate severe degenerative joint disease, were obtained using a wide-field FLIM system with $\mu \mathrm{J}$ excitation pulses at $410 \mathrm{~nm}$ using a GOI operating at $5 \mathrm{kHz}$ (Kentech Instruments Ltd). They clearly contrast the bone, the degenerated cartilage and the subchondral cyst and suggest that FLIM endoscopy may one day provide a useful clinical tool for diagnosis of degenerative joint disease.

We have also applied this wide-field FLIM system to ex vivo human breast tissue and have previously observed contrast between normal and malignant tissue in fixed tissue sections [41]. Figure 6 shows recent FLIM images of unstained frozen sections of human breast tissue featuring a benign fibroadenoma and an invasive ductal carcinoma, together with the corresponding H\&E stained sections. The intrinsic contrast observed between normal tissue and tumours again suggests potential for optical biopsy as a clinical diagnostic.

Figure 7 shows one further example of the potential of FLIM for diagnostic imaging. The FLIM map shown is of an unstained fixed section of rat artery with an atherosclerotic plaque. This sample was excited with $\mathrm{J}$ excitation pulses at $412 \mathrm{~nm}$ using the same wide-field GOI-based FLIM system as for figures 5 and 6 . The atherosclerotic plaque is clearly contrasted from 


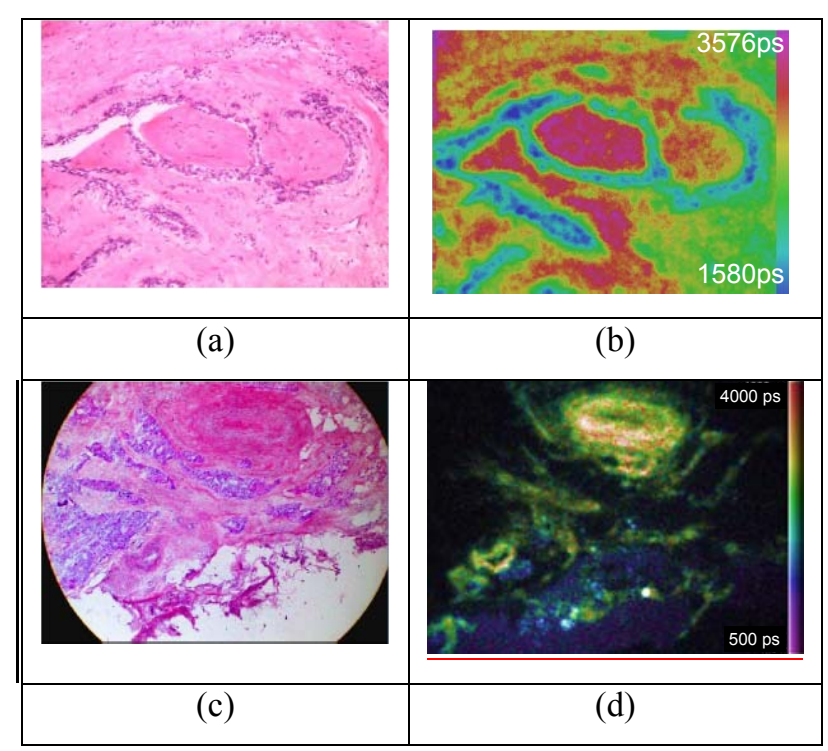

Figure 6. (a, c) H\&E stained sections and $(b, d)$ wide-field FLIM images of unstained frozen sections of $(a, b)$ benign fibroadenoma and $(c, d)$ invasive ductal carcinoma in ex vivo human breast tissue.

the normal arterial wall and this first result will be followed by a more thorough investigation. We note that non-imaging time-resolved studies of arterial plaques have reported lifetime contrast shows increased capability for identification and classification of atherosclerotic plaques, e.g. [42]. This provides further motivation to develop practical in vivo FLIM instrumentation.

\section{Outlook for FLIM of tissue}

It is clear that FLIM is able to provide contrast between different types or states of biological tissue. To realise the biomedical potential of FLIM applied to tissue autofluorescence, it is necessary to identify the specific applications for which it provides useful diagnostic contrast, to understand the origin of this intrinsic contrast and to develop practical FLIM systems for in vivo imaging, including video rate and endoscopic systems. It is important to appreciate that FLIM is just one facet of fluorescence imaging and any practical application should benefit from a careful consideration of other forms of contrast including spectral (excitation and emission wavelengths) and polarisation discrimination. It is clear that the more "dimensions" into which fluorescence is resolved, the greater is the opportunity of contrast - although this enhanced opportunity will usually be accompanied by an increase in experimental complexity and data acquisition time. For a practical FLIM instrument, the data acquisition rate will probably be limited by considerations of motion artifacts, computer data bus speeds, size and sensitivity of available imaging sensors and the time taken to process the data and provide a

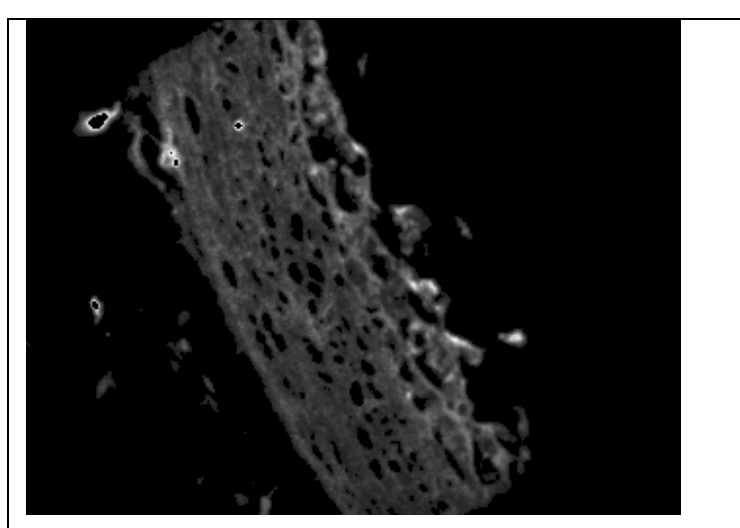

(a)

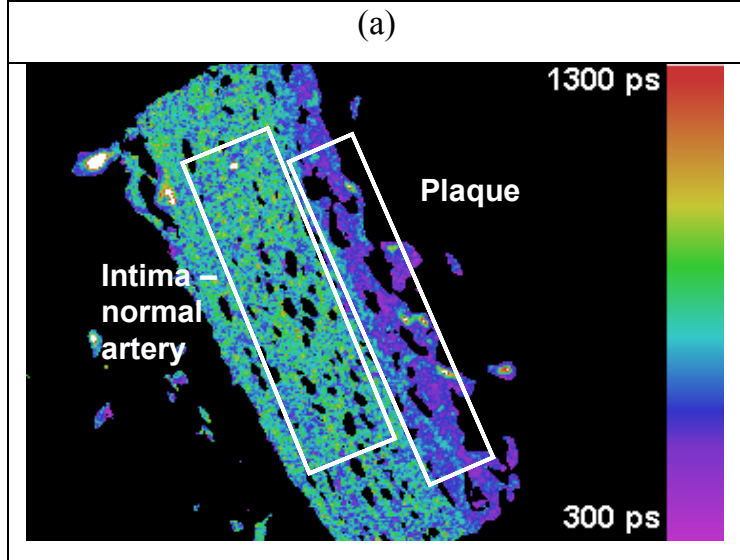

(b)

Figure 7. (a) wide-field fluorescence image and (b) wide-field FLIM image of unstained section of rabbit artery with atherosclerotic plaque

useful output. Given an effective upper limit on data rate, it should be possible to optimise the way in which the fluorescence is resolved in order to maximise the information rate of the instrument to permit the best clinical diagnosis to be made. We have previously investigated the combination of spectrally-resolved imaging with FLIM using a two-channel spectral imager (Optical Insights, Inc.) that uses a dichroic beamsplitter to divide the incoming fluorescence image into a pair of subimages that are spatially identical but which correspond to different spectral pass-bands. This approach, which has enabled us to demonstrate that the fluorescence lifetime can change as a function of the emission detection wavelength range [29], should permit the design of fluorescence imaging instrumentation that can be more specific without increasing the data rate. 


\section{Endoscopic FLIM}

The wide-field FLIM technology described here may be applied to almost any wide-field imaging instruments including endoscopes. To date we are aware of only one report of FLIM endoscopy applied to biological tissue, which utilised frequency domain instrumentation [43], although several non-imaging point measurement instruments have been developed for in vivo application, e.g. [39, 40]. We have made preliminary investigations of time-domain FLIM

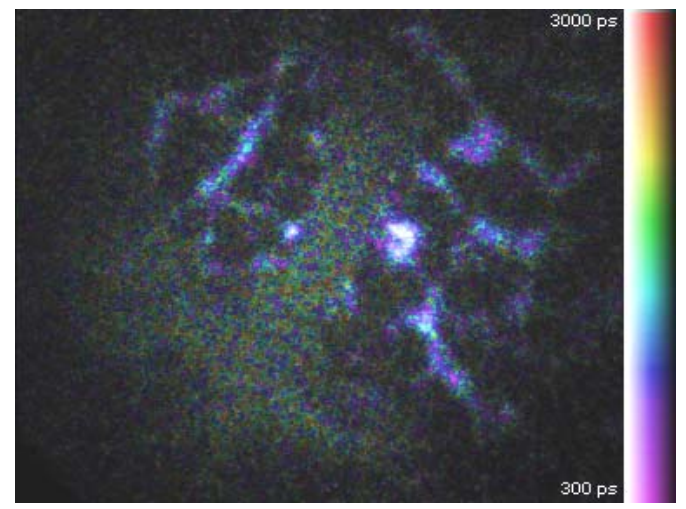

(a)

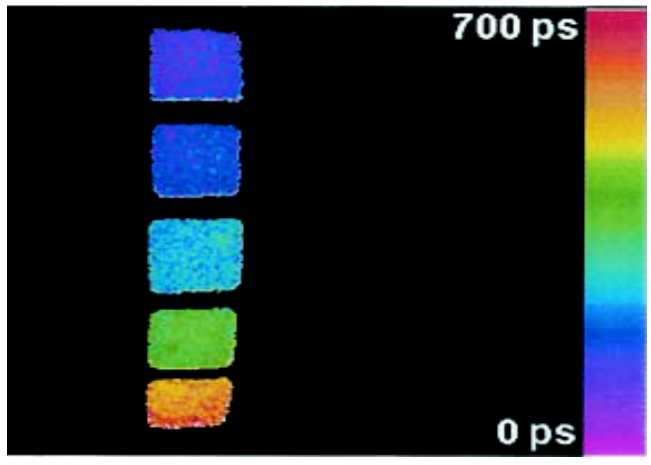

(b)

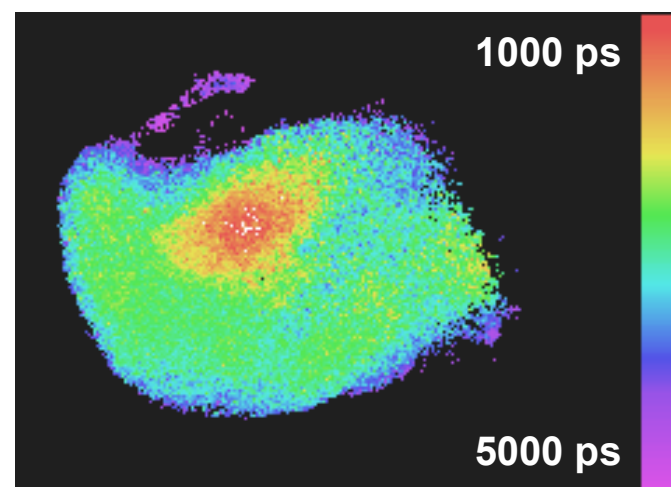

(c)

Figure 8. Endoscopic wide-field FLIM images of (a) unstained section of human bone and dried blood (b) multi-well plate array of DASPI solutions of varying viscosity and (c) unstained rabbit knee joint with bone and tendon. with rigid arthroscopes and with flexible fibre bundle-based endoscopes. Figure 8(a) shows a preliminary FLIM image of a fixed unstained section (human) bone and dried blood obtained through a rigid arthroscope. In principle this approach should produce high resolution FLIM images, as long as there is adequate signal detected. In this particular experiment, the excitation source was a $1 \mathrm{~mW}$ average power picosecond pulsed laser diode at $405 \mathrm{~nm}$. This power level is too low for a practical instrument but the experiment demonstrates that clinical FLIM may become practical with the advent of more powerful portable diode-based ultrafast laser technology. Figures $8(\mathrm{~b}, \mathrm{c})$ are wide-field FLIM images acquired through a flexible endoscope incorporating a $2 \mathrm{~m}$ long bundle of multimode step-index optical fibres [2]. It will be seen that clear contrast is observed with the multi-well plate dye samples and that intrinsic FLIM contrast from autofluorescence is also achievable.

There are still significant issues to be overcome in order to make clinical FLIM practically useful. As well as the need for suitable excitation sources, these include efficient coupling and transmission through the endoscope optical system and appropriate techniques to sample and analyse the complex fluorescence decay profiles presented by most biological tissues. Nevertheless, our early results indicate that FLIM can potentially contrast different states of tissue components such as collagen and elastin. As well as indicating disease, this technology could also be used to provide a real-time monitor of tissue during (minimally invasive) surgery.

\section{Conclusions}

The recently available combinations of relatively low-cost and user-friendly, tunable, ultrafast laser sources and time-resolved detection systems have generated a significant increase in the uptake of FLIM. Today most users are perhaps attracted to FLIM by the relatively straightforward way in which it can be added to multiphoton microscopes and its potential to enhance FRET experiments. In the future it is likely to become much more widespread as the key technological components become cheaper and simpler. It is likely that there will be a number of different commercially available implementations, exploiting time-domain and frequency-domain technology, adapted for specific applications - and markets. For many of these, time-resolved fluorescence imaging will be just a component of multi-parameter fluorescence imaging instruments that provide unprecedented specificity. For now there is much scope to work with research-orientated FLIM instrumentation to identify the most promising applications for which optimised lower cost technology may be 
developed. The results presented here drive our work to investigate optimum strategies to acquire and interpret FLIM data, to catalogue autofluorescence lifetime contrast "signatures" over extended spectral and temporal ranges and to explain the biological and physiological origin of the observed FLIM contrast. We also have an increasing interest in developing FLIM assay technologies, e.g. [44].

\section{Acknowledgements}

We gratefully acknowledge support for this work from the UK Biotechnology and Biological Sciences Research Council (BBSRC), the Engineering and Physical Sciences Research Council (EPSRC), the Department of Trade and Industry (DTI) through a Beacon Award and the Wellcome Trust through a Showcase Award.

\section{References}

$1 \quad$ J. R. Lakowicz, Principles of Fluorescence Spectroscopy, $2^{\text {nd }}$ ed. ,Kluwer Academic/Plenum Publishers, New York, 1999

2 J. Siegel, D. S. Elson, S. E. D. Webb, K. C. Benny Lee, A. Viandas, G. L. Gambaruto, S. LévêqueFort, M. J. Lever, P. J. Tadrous, G. W. H. Stamp, A. L. Wallace, A. Sandison, T. Watson, P. M. W. French, and F. Alvarez, Studying biological tissue with fluorescence lifetime imaging: microscopy, endoscopy, and complex decay profiles, Appl. Opt., 2003, 42, 2995-3004

3 Fluorescence Lifetime Imaging for Biomedicine, invited talk presented by Paul French at the 10th Congress of the European Society for Photobiology, Vienna 2003

4 R. Cubeddu, D. Comelli, C. D'Andrea, P. Taroni, G. Valentini, Time-resolved fluorescence imaging in biology and medicine, Journal of Physics D-Applied Physics, 2002, 35, R61-R76

5 F. S. Wouters, P. J. Verveer, and P. I. H. Bastiaens, Imaging biochemistry inside cells, Trends in Cell Biology. 2001, 11, 203-211

6 P. J. Tadrous, Methods for imaging the structure and function of living tissues and cells: 2 . Fluorescence lifetime imaging, Journal of Pathology, 2000, 191(3), 229-234

7 P. I. H. Bastiaens and A. Squire, Fluorescence lifetime imaging microscopy: spatial resolution of biochemical processes in the cell, Trends in Cell Biology, 1999,. 9, 48--52

8 S. Andersson-Engels, J. Johansson and S. Svanberg, Medical Diagnostic System Based on Simultaneous Multispectral Fluorescence Imaging, Appl. Opt., 1994, 33, 8022-8029.

9 B. B. Das, F. Liu, and R. R. Alfano, Time-resolved fluorescence and photon migration studies in biomedical and model random media, Reports on Progress in Physics, 1997, 60, 227-292

10 D. V. O'Connor and D. Phillips, Time correlated single photon counting, Academic Press, London 1984
11 I. Bugiel, K. König, and H. Wabnitz, Investigation of cells by fluorescence laser scanning microscopy with subnanosecond time resolution, Lasers in the Life Sciences, 1989, 3, 47-53

12 M. Kress, T. Meier, R. Steiner, F. Dolp, R. Erdmann, U. Ortmann, and A. Rück, Time-resolved microspectrofluorometry and fluorescence lifetime imaging of photosensitizers using picosecond pulsed diode lasers in laser scanning microscopes, $J$. Biomed. Opt., 2003, 8, 26-32

13 C. G. Morgan, A. C. Mitchell, and J. G. Murray, Nanosecond time-resolved fluorescence microscopy: principles and practice, Trans. Roy. Microsc. Soc., 1990, 1, 463-466

14 T. W. J Gadella, T. M Jovin, \& R. M. Clegg, Fluorescence lifetime imaging microscopy (FLIM) spatial resolution of structures on the nanosecond timescale, Biophys. Chem., 1993, 48, 221-239

15 K. Carlsson and A. Liljeborg, Simultaneous confocal lifetime imaging of multiple fluorophores using the intensity-modulated multiple-wavelength scanning (IMS) technique, J. Microscopy, 1998, 191, 119-127

16 P. T. C So, T. French, W. M. Yu, K. Berland, C. Y.; Dong, E. Gratton, Time-resolved fluorescence microscopy using two photon excitation, Bioimaging 1995, 3, 49-63

17 T. Ng, A. Squire, G. Hansra, F. Bornancin, C. Prevostel, A. Hanby, W. Harris, D. Barnes, S. Schmidt, H. Mellor, P. I. H. Bastiaens, and P. J. Parker, Imaging protein kinase $\mathrm{C}$ alpha activation in cells, Science, 1999, 283, 2085-2089

18 K. C. B. Lee, J. Siegel, S. E. D. Webb, S. LevequeFort, M. J. Cole, R. Jones, K. Dowling, M. J. Lever, and P. M. W. French, Application of the stretched exponential function to fluorescence lifetime imaging, Biophysical J., 2001, 81, 1265-1274

19 K. Dowling, M. J. Dayel, M. J. Lever, P. M. W. French, J. D. Hares, and A. K. L. DymokeBradshaw, Fluorescence lifetime imaging with picosecond resolution for biomedical applications, Opt. Lett., 1998, 23, 810-812

20 R. Jones, K. Dowling, M. J. Cole, D. ParsonsKaravassilis, M. J. Lever, P. M. W. French, J. D. Hares, and A. K. L. Dymoke- Bradshaw, Fluorescence lifetime imaging using a diodepumped all-solid-state laser system, Electron. Lett., 1999, 35, 256-257

21 D. S. Elson, J. Siegel, S. E. D. Webb, S. LévêqueFort, M. J. Lever, P. M. W. French, K. Lauritsen, M. Wahl, R. Erdmann, Fluorescence lifetime system for microscopy and multi-well plate imaging using a blue picosecond diode laser, Opt. Lett., 2002, 27, 1409-1411

22 J. R. Alcala, E. Gratton, and D. M. Jameson, A Multifrequency Phase Fluorometer Using the Harmonic Content of a Mode-Locked Laser, Analytical Instrumentation., 1985 14, 225-250

23 A. Squire, P. J. Verveer, and P. I. H. Bastiaens, Multiple frequency fluorescence lifetime imaging microscopy, Journal of Microscopy, 2000, 197, 136--149

24 A. C. Mitchell, J. E. Wall, J. G. Murray, and C. G. Morgan, Measurement of nanosecond time-resolved fluorescence with a directly gated interline CCD camera, Journal of Microscopy, 2002, 206, 233-238 
25 M. Petran, M. Hadravsky, M. D. Egger, and R. Galambos, Tandem scanning reflected light microscope, J. Opt. Soc. of Am., 1968, 58, 661-664

26 J.Bewersdorf, R. Pick, \& S. W. Hell, Multifocal multiphoton microscopy, Opt. Lett., 1998, 23, 655657

27 M. A. A. Neil, R. Juškaitis \& T. Wilson, Method of obtaining optical sectioning by using structured light in a conventional microscope, Opt. Lett., 1997, 22, 1905-1907

28 M. J. Cole, J. Siegel, S. E. D. Webb, R. Jones, K. Dowling, M. J. Dayel, D. Parsons-Karavassilis, P. M. W. French, M. J. Lever, L. O. D. Sucharov, M. A. A. Neil, R. Juškaitis, and T. Wilson, Timedomain whole-field fluorescence lifetime imaging with optical sectioning, J. Microscopy, 2001, 203, 246-257

29 J. Siegel D. S. Elson,., S. E. D. Webb, D. ParsonsKaravassilis, Leveque-Fort, S., M. J. Cole, M. J. Lever, P. M. W. French,, M. A. A. Neil, R. Juškaitis, L. O. D. Sucharov and T. Wilson, Wholefield 5-D fluorescence microscopy combining lifetime and spectral resolution with optical sectioning, Opt. Lett., 2001, 26, 1338-1340

30 K. Suhling, J. Siegel, D. Phillips, P. M. W. French, S. Lévêque-Fort, S. E. D. Webb, and D. M. Davis, Imaging the environment of green fluorescent protein, Biophysical J., 2002, 83, 3589-3595

31 D. Toptygin, R. S. Savtchenko, N. D. Meadow, S. Roseman, and L. Brand, Effect of the solvent refractive index on the excited-state lifetime of a single tryptophan residue in a protein, $J$. Chem. Phys. B, 2002, 106, 3724-3734

32 D. M. Davis, Assembly of the immunological synapse for T cells and NK cells, Trends Immunol., 2002, 23, 356-363

33 B. Treanor, P. Lanigan, K. Suhling, M Neil, D. Phillips D. M. Davis, and P. M.W. French, Probing the microenvironment of proteins by Fluorescence Lifetime Imaging, Submitted to the Biophysical Journal

34 L. Stryer \& R. P. Haugland, Energy transfer: a spectroscopic ruler, Proc Natl Acad Sci USA, 1967, $58719-726$

35 E. A. Jares-Erijman \& T. M. Jovin, FRET Imaging, Nat. Biotechnol., 2003, 21, 1387-1395

36 A. G. Harpur, F.S. Wouters and P. I. Bastiaens, Imaging FRET between spectrally similar GFP molecules in single cells, Nat. Biotechnol., 2001, 19, 167-169.

37 R. RichardsKortum and E. SevickMuraca, Quantitative optical spectroscopy for tissue diagnosis, Annual Review of Physical Chemistry, 1996. 47: 555-606.

38 G. A. Wagnieres, W. M. Star, and B. C. Wilson, In vivo fluorescence spectroscopy and imaging for oncological applications, Photochem. and Photobiol., 1998. 68(5): 603-632.

39 T. Glanzmann, J. P. Ballini, H. van den Bergh, and G. Wagnieres, Time-resolved spectrofluorometer for clinical tissue characterization during endoscopy, Rev. Sci. Instrum, 1999, 70, 4067-4077

40 J. D. Pitts and M. A. Mycek, Design and development of a rapid acquisition laser-based fluorometer with simultaneous spectral and temporal resolution, Rev. Sci. Instrum., 2001, 72, 3061-3072
41 P. J. Tadrous, J. Siegel, P. M. French, S. Shousha, E. N. Lalani, and G. W. Stamp, Fluorescence lifetime imaging of unstained tissues: early results in human breast cancer, J. Path., 2003, 199, 309317

42 L. Marcu, M. C. Fishbein, J. M. I. Maarek, and W. S. Grundfest, Discrimination of human coronary artery atherosclerotic lipid- rich lesions by timeresolved laser-induced fluorescence spectroscopy, Arterioscler. Thromb. Vasc. Biol, 2001,. 21, 12441250

43 J. Mizeret, T. Stepinac, M. Hansroul, A. Studzinski, $H$. van den Bergh, and G. Wagnières, Instrumentation for real-time fluorescence lifetime imaging in endoscopy, Rev. Sci. Instrum., 1999, 70, 4689

44 G. Valentini, C. D'Andrea, D. Comelli, A. Pifferi, P. Taroni, A. Torricelli, R. Cubeddu, C. Battaglia, C. Consolandi, G. Salani, L. Rossi-Bernardi, and G. De Bellis, Time-resolved DNA-microarray reading by an intensified CCD for ultimate sensitivity, Opt. Lett., 2000, 25, 1648-1650 